Journal of Applied AnALysis

Vol. 11, No. 1 (2005), pp. 113-132

\title{
ORTHOGONAL BASES FOR SPACES OF COMPLEX SPHERICAL HARMONICS
}

\author{
V. A. MENEGATTO and C. P. OLIVEIRA \\ Received April 23, 2003 and, in revised form, February 11, 2004
}

\begin{abstract}
This paper proposes an inductive method to construct bases for spaces of spherical harmonics over the unit sphere $\Omega_{2 q}$ of $\mathbb{C}^{q}$. The bases are shown to have many interesting properties, among them orthogonality with respect to the inner product of $L^{2}\left(\Omega_{2 q}\right)$. As a bypass, we study the inner product $[f, g]=f(\bar{D})(\overline{g(z)})(0)$ over the space $\mathbb{P}\left(\mathbb{C}^{q}\right)$ of polynomials in the variables $z, \bar{z} \in \mathbb{C}^{q}$, in which $f(\bar{D})$ is the differential operator with symbol $f(\bar{z})$. On the spaces of spherical harmonics, it is shown that the inner product $[\cdot, \cdot]$ reduces to a multiple of the $L^{2}\left(\Omega_{2 q}\right)$ inner product. Bi-orthogonality in $\left(\mathbb{P}\left(\mathbb{C}^{q}\right),[\cdot, \cdot]\right)$ is fully investigated.
\end{abstract}

\section{Introduction}

This paper considers spaces of polynomials in the variables $z$ and $\bar{z}$ of $\mathbb{C}^{q}$, $q \geq 1$. The unitary space $\mathbb{C}^{q}$ is assumed to be accompanied with its usual inner product

$$
\langle z, w\rangle:=z_{1} \overline{w_{1}}+z_{2} \overline{w_{2}}+\cdots+z_{q} \overline{w_{q}}, \quad z, w \in \mathbb{C}^{q},
$$

2000 Mathematics Subject Classification. 33C55, 33C50, 42C05, 31B05, 33C45, 30C10.

Key words and phrases. Spherical harmonics, sphere, orthogonal basis, generating function, addition formula, Funk-Hecke formula.

ISSN 1425-6908（C) Heldermann Verlag. 
where we are writing $z=\left(z_{1}, z_{2}, \ldots, z_{q}\right)$ and $w=\left(w_{1}, w_{2}, \ldots, w_{q}\right)$. The major polynomial space considered here is $\mathbb{P}\left(\mathbb{C}^{q}\right)$, the unitary space of polynomials in the independent variables $z$ and $\bar{z}$ of $\mathbb{C}^{q}$. Elements of this space can be written in the form

$$
p(z):=p(z, \bar{z})=\sum_{|\alpha| \leq m} \sum_{|\beta| \leq n} p_{\alpha, \beta} z^{\alpha} \bar{z}^{\beta}, \quad p_{\alpha, \beta} \in \mathbb{C}, \quad \alpha, \beta \in \mathbb{Z}_{+}^{q},
$$

for nonnegative integers $m$ and $n$, where standard multi-index notation is in force. The subspace of $\mathbb{P}\left(\mathbb{C}^{q}\right)$ composed of polynomials that are homogeneous of degree $m$ in $z$ and of degree $n$ in $\bar{z}$ will be denoted by $\mathbb{P}_{m, n}\left(\mathbb{C}^{q}\right)$. The dimension of $\mathbb{P}_{m, n}\left(\mathbb{C}^{q}\right)$ is given by $([2, \mathrm{p} .17])$

$$
\delta(q, m, n):=\left(\begin{array}{c}
m+q-1 \\
q-1
\end{array}\right)\left(\begin{array}{c}
n+q-1 \\
q-1
\end{array}\right) .
$$

The subspace of $\mathbb{P}_{m, n}\left(\mathbb{C}^{q}\right)$ composed of harmonic elements, that is, elements that are in the kernel of the complex Laplacian

$$
\Delta_{2 q}:=4 \sum_{j=1}^{q} \frac{\partial^{2}}{\partial z_{j} \partial \overline{z_{j}}}
$$

will be denoted by $\mathbb{H}_{m, n}\left(\mathbb{C}^{q}\right)$. Elements of this space play the role played by the solid harmonics in analysis on real spheres.

Next, we introduce spaces of polynomials restricted to the unit sphere

$$
\Omega_{2 q}:=\left\{z \in \mathbb{C}^{q}:\langle z, z\rangle=1\right\} .
$$

The symbol $\mathcal{P}_{m, n}\left(\Omega_{2 q}\right)$ will stand for the space obtained from $\mathbb{P}_{m, n}\left(\mathbb{C}^{q}\right)$ by restricting its elements to $\Omega_{2 q}$. Finally, $\mathcal{H}_{m, n}\left(\Omega_{2 q}\right)$ will denote the space of complex spherical harmonics of degree $m$ in $z$ and degree $n$ in $\bar{z}$, that is, the set of restrictions of elements of $\mathbb{H}_{m, n}\left(\mathbb{C}^{q}\right)$ to $\Omega_{2 q}$. The space $\mathcal{H}_{m, n}\left(\Omega_{2 q}\right)$ has dimension $d(q, m, n)$ given by $([2, \mathrm{p} .17])$

$$
\begin{gathered}
d(q, m, n)=\delta(q, m, n)-\delta(q, m-1, n-1), \quad m, n \neq 0, \\
(q, m, 0)=\delta(q, m, 0), \quad \text { and } \quad \delta(q, 0, n)=\delta(q, 0, n) .
\end{gathered}
$$

This paper was motivated by the following three results: the orthogonal decomposition $([2])$

$$
\mathcal{P}_{m, n}\left(\Omega_{2 q}\right)=\bigoplus_{j=0}^{m \wedge n} \mathcal{H}_{m-j, n-j}\left(\Omega_{2 q}\right),
$$

the dimension formula ([2], [8])

$$
d(q, m, n)=\sum_{k=0}^{m} \sum_{l=0}^{n} d(q-1, k, l), \quad q \geq 2,
$$


and the fact that some elements of $\mathcal{H}_{m, n}\left(\Omega_{2 q}\right)$ can be constructed from given elements in $\mathcal{H}_{m-k, n-l}\left(\Omega_{2 q}\right), k<m, l<n$, by multiplying them by special elements of $\mathbb{H}_{k, l}\left(\mathbb{C}^{q}\right)$ (see proof of Theorem 5.1 in [3]).

Looking at the real version of (1.8) in either $[1$, p. 76$]$ or $[9$, p. 139] one observes that the proof there requires a special inner product on spaces of homogeneous polynomials. In the first half of the paper, we endow our polynomial spaces with the following similar inner product

$$
[f, g]:=[f, g]_{q}:=f(\bar{D})(\overline{g(z)})(0), \quad f, g \in \mathbb{P}\left(\mathbb{C}^{q}\right),
$$

in which

$$
\bar{D}:=\left(\frac{\partial}{\partial \overline{z_{1}}}, \frac{\partial}{\partial \overline{z_{2}}}, \cdots, \frac{\partial}{\partial \overline{z_{q}}}\right),
$$

and extract a number of interesting properties. Among them, we show that there is a positive constant $C$, depending on $m, n$ and $q$, such that

$$
[f, g]=C\langle f, g\rangle_{2}, \quad f, g \in \mathcal{H}_{m, n}\left(\Omega_{2 q}\right) .
$$

The inner product in the right-hand side of (1.12) is the usual one in $L^{2}\left(\Omega_{2 q}\right)$, that is,

$$
\langle f, g\rangle_{2}:=\int_{\Omega_{2 q}} f(z) \overline{g(z)} d \sigma_{q}(z), \quad f, g \in L^{2}\left(\Omega_{2 q}\right),
$$

where $\sigma_{q}$ is a positive Borel measure invariant by isometries of $C^{q}$ and uniquely determined by the condition

$$
\sigma_{q}\left(\Omega_{2 q}\right)=\frac{2 \pi^{q}}{(q-1) !} .
$$

The other properties we obtain are related to the Funk-Hecke formula ([5], [6]) and with properties of bi-orthogonal systems in the polynomial spaces endowed with the inner product in (1.10). All the results mentioned above form the contents of Sections 2 and 3.

Formula (1.9) suggests that one should be able to construct a basis for $\mathcal{H}_{m, n}\left(\Omega_{2 q}\right)$ from given bases for the spaces $\mathcal{H}_{k, l}\left(\Omega_{2 q-2}\right), k=0,1, \ldots, m$, $l=0,1, \ldots, n$. We prove this is the case using the special polynomials introduced in [3, p. 3] as a generating function. In addition, we discuss orthogonality and representing properties that are implied by the result, completing the list of results forming Section 4.

\section{The inner product $[\cdot, \cdot]$}

To begin this section, we observe that the spaces $\mathcal{H}_{m, n}\left(\Omega_{2 q}\right)$ are pairwise orthogonal with respect to the inner product $\langle\cdot, \cdot\rangle_{2}([3])$. Throughout the paper, orthogonality will always refer to this inner product. 
If $\mathcal{O}(2 q)$ is the group of isometries of $\mathbb{C}^{q}$ that fix the origin then $\sigma_{q}$ is $\mathcal{O}(2 q)$-invariant in the following sense: $\sigma_{q}(\rho B)=\sigma_{q}(B)$ if $\rho \in \mathcal{O}(2 q)$ and $B$ is a Borel subset of $\Omega_{2 q}$. As a consequence, the following invariance property holds:

$$
\langle f \circ \rho, g \circ \rho\rangle_{2}=\langle f, g\rangle_{2}, \quad f, g \in L^{2}\left(\Omega_{2 q}\right), \quad \rho \in \mathcal{O}(2 q) .
$$

The following well-known result establishes the $\mathcal{O}(2 q)$-invariance of complex spherical harmonics.

Lemma 2.1. The space $\mathcal{H}_{m, n}\left(\Omega_{2 q}\right)$ is $\mathcal{O}(2 q)$-invariant, that is, if $f \in$ $\mathcal{H}_{m, n}\left(\Omega_{2 q}\right)$ and $\rho \in \mathcal{O}(2 q)$ then $f \circ \rho \in \mathcal{H}_{m, n}\left(\Omega_{2 q}\right)$.

Proof. It will be left to the reader.

Next, we return to formula (1.10).

Lemma 2.2. Formula (1.10) defines an inner product in $\mathbb{P}\left(\mathbb{C}^{q}\right)$.

Proof. It is very easy to see from the definitions that if $(i, j) \neq(k, l)$ then the spaces $\mathbb{P}_{i, j}\left(\mathbb{C}^{q}\right)$ and $\mathbb{P}_{k, l}\left(\mathbb{C}^{q}\right)$ are orthogonal with respect to $[\cdot, \cdot]$. In particular, we have

$$
\left[z^{\alpha} \bar{z}^{\beta}, z^{\gamma} \bar{z}^{\delta}\right]= \begin{cases}\alpha ! \beta !, & (\alpha, \beta)=(\gamma, \delta) \\ 0, & (\alpha, \beta) \neq(\gamma, \delta)\end{cases}
$$

Now, let $f, g \in \mathbb{P}\left(\mathbb{C}^{q}\right)$. There are pairs of indices $(k, l)$ and $(m, n)$ in $\mathbb{Z}_{+}^{2}$ such that

$$
\begin{aligned}
f(z)=\sum_{i=0}^{k} \sum_{j=0}^{l} f_{i, j}(z), \quad g(z)=\sum_{\substack{\mu=0 \\
f_{i, j} \in 0}}^{m} \sum_{\mathbb{P}_{i, j}\left(\mathbb{C}^{q}\right), \quad g_{\mu, \nu} \in \mathbb{P}_{\mu, \nu}\left(\mathbb{C}^{q}\right) .} .
\end{aligned}
$$

Hence,

$$
[f, g]=\sum_{i=0}^{k} \sum_{j=0}^{l} \sum_{\mu=0}^{m} \sum_{\nu=0}^{n}\left[f_{i, j}, g_{\mu, \nu}\right]=\sum_{\mu=0}^{k \wedge m} \sum_{\nu=0}^{l \wedge n}\left[f_{\mu, \nu}, g_{\mu, \nu}\right] .
$$

Expanding $f_{\mu, \nu}$ and $g_{\mu, \nu}$ in the form

$$
f_{\mu, \nu}(z)=\sum_{|\alpha|=\mu} \sum_{|\beta|=\nu} a_{\alpha, \beta} z^{\alpha} \bar{z}^{\beta}, \quad g_{\mu, \nu}(z)=\sum_{|\gamma|=\mu|\delta|=\nu} \sum_{a_{\alpha, \beta}, b_{\gamma, \delta} \in \mathbb{C},} b_{\gamma, \delta} z^{\gamma} \bar{z}^{\delta},
$$

we finally deduce that

$$
[f, g]=\sum_{\mu=0}^{k \wedge m} \sum_{\nu=0}^{l \wedge n} \sum_{|\alpha|=\mu} \sum_{|\beta|=\nu} \alpha ! \beta ! a_{\alpha, \beta} \overline{b_{\alpha, \beta}} .
$$


Using this representation, it is now easy to verify that $[\cdot, \cdot]$ defines an inner product in the space $\mathbb{P}\left(\mathbb{C}^{q}\right)$.

As an example, we observe that the set

$$
\bigcup_{m, n \in \mathbb{Z}_{+}}\left\{\frac{z^{\alpha}}{\sqrt{\alpha !}} \frac{\bar{z}^{\beta}}{\sqrt{\beta !}}:|\alpha|=m,|\beta|=n\right\}
$$

is an orthonormal basis for $\left(\mathbb{P}\left(\mathbb{C}^{q}\right),[\cdot, \cdot]\right)$. Another remark at this time is that formula (1.10) reduces to

$$
[f, g]=f(\bar{D})(\overline{g(z)}),
$$

when the space $\mathbb{P}\left(\mathbb{C}^{q}\right)$ is replaced with its subspace $\mathbb{P}_{m, n}\left(\mathbb{C}^{q}\right)$. At last, we observe that formula (2.6) is a complex extension of that appearing in Theorem 5.14 in [1].

Lemma 2.3. The inner product $[\cdot, \cdot]$ possesses the following invariance property

$$
[f \circ \rho, f \circ \rho]=[f, f], \quad f \in \mathbb{P}_{m, n}(\mathbb{C}), \quad \rho \in \mathcal{O}(2 q) .
$$

Proof. Since every element of $\mathbb{P}_{m, n}\left(\mathbb{C}^{q}\right)$ is a linear combination of elements of the form $z^{\alpha} \bar{z}^{\beta}$, it suffices to verify the formula in the statement of the lemma for elements of this type. However, since

$$
\left[z^{\alpha} \bar{z}^{\beta} \circ \rho, z^{\gamma} \bar{z}^{\delta} \circ \rho\right]=\left[z^{\alpha} \circ \rho, z^{\gamma} \circ \rho\right]\left[\bar{z}^{\beta} \circ \rho, \bar{z}^{\delta} \circ \rho\right], \quad \rho \in \mathcal{O}(2 q),
$$

it suffices to prove the formula in the case in which $f(z)=z^{\alpha}$ and $g(z)=z^{\gamma}$, $|\alpha|=|\gamma|$, and in the conjugate case of this one. Let $\rho \in \mathcal{O}(2 q)$ be described as

$$
\rho(z)=\left(\sum_{j=1}^{q} a_{1 j} z_{j}, \sum_{j=1}^{q} a_{2 j} z_{j}, \ldots, \sum_{j=1}^{q} a_{q j} z_{j}\right), \quad a_{l j} \in \mathbb{C}, \quad z \in \mathbb{C}^{q} .
$$

If $f$ and $g$ are as above then the formula to be proven is

$$
[f \circ \rho, g \circ \rho]=\prod_{l=1}^{q} D_{l}^{\alpha_{l}} \overline{\rho(z)^{\gamma}},
$$

where

$$
D_{l}:=a_{l 1} \frac{\partial}{\partial \bar{z}_{1}}+a_{l 2} \frac{\partial}{\partial \bar{z}_{2}}+\cdots+a_{l q} \frac{\partial}{\partial \bar{z}_{q}}, \quad l=1,2, \ldots, q .
$$

First consider the case $\alpha=\gamma$. Using the relation

$$
\sum_{k=1}^{q} \overline{a_{j k}} a_{l k}= \begin{cases}0 & \text { if } l \neq j \\ 1 & \text { if } l=j\end{cases}
$$


it is easily seen that

$$
D_{l}^{\alpha_{l}} \overline{\rho(z)}^{\alpha}=\alpha_{l} ! \overline{\rho(z)}^{\alpha-\alpha_{l} \varepsilon_{l}}, \quad l=1,2, \ldots, q .
$$

It follows that $[f \circ \rho, g \circ \rho]=\alpha !=\left[z^{\alpha}, z^{\alpha}\right]$. If $\alpha \neq \gamma$, we can assume without loss of generality, that $\alpha_{j}>\gamma_{j}$ for some $j$. In this case, $D_{j}^{\alpha_{j}} \overline{\rho(z)}^{\gamma}=0$, that is, $[f \circ \rho, g \circ \rho]=0=\left[z^{\alpha}, z^{\gamma}\right]$. The conjugate case is dealt with in a similar manner.

Next, we employ the vector space isomorphism

$$
\left.f \in \mathbb{H}_{m, n}\left(\mathbb{C}^{q}\right) \longmapsto f\right|_{\Omega_{2 q}} \in \mathcal{H}_{m, n}\left(\Omega_{2 q}\right)
$$

to bring the inner product (1.10) into the space $\mathcal{H}_{m, n}\left(\Omega_{2 q}\right)$. If $f \in \mathcal{H}_{m, n}\left(\Omega_{2 q}\right)$ write $\widehat{f}$ to denote the unique element of $\mathbb{H}_{m, n}\left(\mathbb{C}^{q}\right)$ such that $\left.\widehat{f}\right|_{\Omega_{2 q}}=f$. Then the formula

$$
[f, g]:=[\widehat{f}, \widehat{g}], \quad f, g \in \mathcal{H}_{m, n}\left(\Omega_{2 q}\right)
$$

defines an inner product in $\mathcal{H}_{m, n}\left(\Omega_{2 q}\right)$.

Theorem 2.7 below will reveal that the spaces $\left(\mathcal{H}_{m, n}\left(\Omega_{2 q}\right),[\cdot, \cdot]\right)$ and $\left(\mathcal{H}_{m, n}\left(\Omega_{2 q}\right),\langle\cdot, \cdot\rangle_{2}\right)$ are isomorphic. The following results will be helpful in proving that theorem. Details about them can be found in [2]. The proof of the first one can also be adapted from results proved in [6, p. 17]. From now on, the symbol $\varepsilon_{j}$ will stand for the vector of $\mathbb{C}^{q}$ having 1 in its $j^{\text {th }}$ component and zeros elsewhere.

Lemma 2.4. If $W$ is a nonzero finite-dimensional $\mathcal{O}(2 q)$-invariant space of continuous functions on $\Omega_{2 q}$ then there exists a unique $f$ in $W \backslash\{0\}$ such that $f \circ \rho=f$, when $\rho \in \mathcal{O}(2 q)$ and $\rho\left(\varepsilon_{q}\right)=\varepsilon_{q}$.

Lemma 2.5. Let $f$ be in $\mathcal{H}_{m, n}\left(\Omega_{2 q}\right)$. The following assertions are equivalent:

i) $f \circ \rho=f$ if $\rho \in \mathcal{O}(2 q)$ and $\rho\left(\varepsilon_{q}\right)=\varepsilon_{q}$;

ii) There exists a complex number $C$ such that

$$
\begin{array}{r}
f(z)=C e^{i(m-n) \theta}\left|\left\langle z, \varepsilon_{q}\right\rangle\right|^{|m-n|} P_{m \wedge n}^{(q-2,|m-n|)}\left(2\left|\left\langle z, \varepsilon_{q}\right\rangle\right|^{2}-1\right), \\
z \in \Omega_{2 q},
\end{array}
$$

in which $\theta$ is an argument of $\left\langle z, \varepsilon_{q}\right\rangle$ in $[0,2 \pi)$.

Proposition 2.6. Let $\mathcal{N}$ be a subspace of $\mathcal{H}_{m, n}\left(\Omega_{2 q}\right)$. If $\mathcal{N}$ is $\mathcal{O}(2 q)$ invariant then either $\mathcal{N}=\{0\}$ or $\mathcal{N}=\mathcal{H}_{m, n}\left(\Omega_{2 q}\right)$. 
Proof. If $\mathcal{N} \neq\{0\}$ then $\mathcal{H}_{m, n}\left(\Omega_{2 q}\right)=\mathcal{N} \oplus \mathcal{N}^{\perp}$, in which $\mathcal{N}^{\perp}$ is the orthogonal complement of $\mathcal{N}$ in $\mathcal{H}_{m, n}\left(\Omega_{2 q}\right)$. Obviously, $\mathcal{N}^{\perp}$ is $\mathcal{O}(2 q)$-invariant. The rest of the proof will show that $\mathcal{N}^{\perp}=\{0\}$. Indeed, if not, we may use Lemma 2.4 to choose $f \in \mathcal{N} \backslash\{0\}$ and $g \in \mathcal{N}^{\perp} \backslash\{0\}$ such that $f \circ \rho=f$ and $g \circ \rho=g$, when $\rho \in \mathcal{O}(2 q)$ and $\rho\left(\varepsilon_{q}\right)=\varepsilon_{q}$. Lemma 2.5 furnishes a complex number $C$ such that $f=C g$. It follows that $f=g=0$, a clear contradiction.

Theorem 2.7. There exists a positive constant $C$, depending on $m, n$ and $q$, such that

$$
[f, g]=C\langle f, g\rangle_{2}, \quad f, g \in \mathcal{H}_{m, n}\left(\Omega_{2 q}\right)
$$

Proof. Since $F:=\left\{f \in \mathcal{H}_{m, n}\left(\Omega_{2 q}\right):\langle f, f\rangle_{2}=1\right\}$ is a compact subset of $\mathcal{H}_{m, n}\left(\Omega_{2 q}\right)$, the continuous function

$$
f \in F \longmapsto[f, f] \in \mathbb{R}
$$

attains its maximum in a point $f_{0}$ of $F$. It follows that,

$$
[f, f] \leq\left[f_{0}, f_{0}\right]\langle f, f\rangle_{2}, \quad f \in \mathcal{H}_{m, n}\left(\Omega_{2 q}\right) .
$$

We will use this information to show that the bilinear form

$$
\varphi: \mathcal{H}_{m, n}\left(\Omega_{2 q}\right) \times \mathcal{H}_{m, n}\left(\Omega_{2 q}\right) \longrightarrow \mathbb{C}
$$

given by

$$
\varphi(f, g)=\left[f_{0}, f_{0}\right]\langle f, g\rangle_{2}-[f, g], \quad f, g \in \mathcal{H}_{m, n}\left(\Omega_{2 q}\right)
$$

is identically zero. Equivalently, we will show that

$$
\mathcal{N}:=\left\{f \in \mathcal{H}_{m, n}\left(\Omega_{2 q}\right): \varphi(f, g)=0, \quad g \in \mathcal{H}_{m, n}\left(\Omega_{2 q}\right)\right\}
$$

is the whole space $\mathcal{H}_{m, n}\left(\Omega_{2 q}\right)$. Since $\mathcal{N}$ is a subspace of $\mathcal{H}_{m, n}\left(\Omega_{2 q}\right)$, Proposition 2.6 tells us that it suffices to show that $\mathcal{N}$ is nonzero and $\mathcal{O}(2 q)$ invariant. Let $\rho \in \mathcal{O}(2 q)$ and $f \in \mathcal{N}$. Due to (2.21), $\varphi$ is positive definite. Hence, we may apply Schwarz's inequality [4, p. 375] to obtain

$$
|\varphi(f \circ \rho, g)|^{2} \leq \varphi(f \circ \rho, f \circ \rho) \varphi(g, g), \quad g \in \mathcal{H}_{m, n}\left(\Omega_{2 q}\right) .
$$

However, Lemma 2.3 and property $(2.1)$ imply that $\varphi(f \circ \rho, f \circ \rho)=\varphi(f, f)=$ 0 . It follows that $f \circ \rho \in \mathcal{N}$. Since a similar argument shows that $\varphi\left(f_{0}, g\right)=0$, $g \in \mathcal{H}_{m, n}\left(\Omega_{2 q}\right)$, it is clear that $\mathcal{N}$ is nonzero.

Corollary 2.8. There exists a positive constant $C$ such that

$$
[f, g]=C\left\langle\left. f\right|_{\Omega_{2 q}},\left.g\right|_{\Omega_{2 q}}\right\rangle_{2}, \quad f, g \in \mathbb{H}_{m, n}\left(\mathbb{C}^{q}\right) .
$$

Next, we compute the constant $C$ in Theorem 2.7. The following lemma is taken from Rudin's book [8, p. 16]. 
Lemma 2.9. For multi-indices $\alpha$ and $\beta$ we have

$$
\int_{\Omega_{2 q}} z^{\alpha} \bar{z}^{\beta} d \sigma_{q}(z)= \begin{cases}0 & \text { if } \alpha \neq \beta \\ \frac{2 \pi^{q} \alpha !}{(|\alpha|+q-1) !} & \text { if } \alpha=\beta .\end{cases}
$$

Take $f(z)=g(z)=z_{1}^{m}{\overline{z_{2}}}^{n}$ in the space $\mathbb{H}_{m, n}\left(\mathbb{C}^{q}\right)$. Formula (2.2) implies that $[f, g]=m ! n$ ! while Lemma 2.9 produces

$$
\langle f, g\rangle_{2}=\frac{2 \pi^{q} m ! n !}{(m+n+q-1) !} .
$$

This proves the following theorem.

Theorem 2.10. The constant $C$ in Theorem 2.7 equals to $(m+n+q-$ $1) !\left(2 \pi^{q}\right)^{-1}$.

We close the section by showing that Theorem 2.7 cannot hold in the bigger space $\mathcal{P}_{m, n}\left(\Omega_{2 q}\right)$. In fact, if $h(z)=z_{1}^{m}{\overline{z_{1}}}^{n}$ then $[h, h]=m ! n$ ! while Lemma 2.9 yields $\langle h, h\rangle_{2}=2 \pi^{q}(m+n) ! /(m+n+q-1)$ !. Now, it is easily seen that the equality $[h, h]=C\langle h, h\rangle_{2}$ holds if and only if $C=$ $m ! n !(m+n+q-1) !\left(2 \pi^{q}\right)^{-1} /(m+n) !$. This is not the value of $C$ we have encountered in Theorem 2.10 .

\section{Bi-orthogonality in $\left(\mathbb{P}\left(\mathbb{C}^{q}\right),[\cdot, \cdot]\right)$}

In this section we investigate orthogonality in the space $\left(\mathbb{P}\left(\mathbb{C}^{q}\right),[\cdot, \cdot]\right)$. We begin with a result related to basic elements of $\left(\mathbb{P}_{m, n}\left(\mathbb{C}^{q}\right),[\cdot, \cdot]\right)$.

Theorem 3.1. Let $\left\{f_{\mu}: \mu=1,2, \ldots, \delta(q, m, n)\right\}$ and $\left\{g_{\nu}: \nu=\right.$ $1,2, \ldots, \delta(q, m, n)\}$ be bases for $\left(\mathbb{P}_{m, n}\left(\mathbb{C}^{q}\right),[\cdot, \cdot]\right)$. If $\left[f_{\mu}, g_{\nu}\right]=0, \mu \neq \nu$ then

$$
\langle z, w\rangle^{m}\langle w, z\rangle^{n}=m ! n ! \sum_{\mu=1}^{\delta(q, m, n)} \frac{f_{\mu}(z) \overline{g_{\mu}(w)}}{\left[f_{\mu}, g_{\mu}\right]}, \quad z, w \in \mathbb{C}^{q} .
$$

Proof. Since $\left\{f_{\mu}: \mu=1,2, \ldots, \delta(q, m, n)\right\}$ is a basis for $\mathbb{P}_{m, n}\left(\mathbb{C}^{q}\right)$, there are polynomials $p_{\mu}, \mu=1,2, \ldots, \delta(q, m, n)$ such that

$$
\langle z, w\rangle^{m}\langle w, z\rangle^{n}=\sum_{\mu=1}^{\delta(q, m, n)} p_{\mu}(w) f_{\mu}(z), \quad z, w \in \mathbb{C}^{q} .
$$

Due to the hypothesis,

$$
\left[\langle\cdot, w\rangle^{m}\langle w, \cdot\rangle^{n}, g_{\nu}\right]=\sum_{\mu=1}^{\delta(q, m, n)} p_{\mu}(w)\left[f_{\mu}, g_{\nu}\right]
$$


ORTHOGONAL BASES FOR SPACES OF COMPLEX SPHERICAL HARMONICS 121

$$
=p_{\nu}(w)\left[f_{\nu}, g_{\nu}\right], \quad \nu=1,2, \ldots, \delta(q, m, n), \quad w \in \mathbb{C}^{q} .
$$

On the other hand, writing $g_{\nu}$ in the form

$$
g_{\nu}(z)=\sum_{|\alpha|=m} \sum_{|\beta|=n} c_{\alpha, \beta} z^{\alpha} \bar{z}^{\beta}
$$

and computing, we obtain

$$
\begin{aligned}
{\left[\langle\cdot, w\rangle^{m}\langle w, \cdot\rangle^{n}, g_{\nu}\right] } & =m ! n ! \sum_{|\gamma|=m} \sum_{|\delta|=n} \sum_{|\alpha|=m} \sum_{|\beta|=n} \frac{\bar{w}^{\gamma}}{\gamma !} \frac{w^{\delta}}{\delta !} \overline{c_{\alpha, \beta}}\left[z^{\gamma} \bar{z}^{\delta}, z^{\alpha} \bar{z}^{\beta}\right] \\
& =m ! n ! \sum_{|\alpha|=m|\beta|=n} \sum_{\overline{c_{\alpha, \beta}}} \bar{w}^{\alpha} w^{\beta} \\
& =m ! n ! \overline{g_{\nu}(w)}, \quad \nu=1,2, \ldots, \delta(m, n) .
\end{aligned}
$$

Thus,

$$
m ! n ! \overline{g_{\nu}(w)}=p_{\nu}(w)\left[f_{\nu}, g_{\nu}\right], \quad \nu=1,2, \ldots, \delta(q, m, n), \quad w \in \mathbb{C}^{q},
$$

and, in particular, since each $g_{\mu}$ is not identically zero, $\left[f_{\mu}, g_{\mu}\right] \neq 0, \mu=$ $1,2, \ldots, \delta(m, n)$. Concluding,

$$
p_{\mu}=m ! n ! \frac{\overline{g_{\mu}}}{\left[f_{\mu}, g_{\mu}\right]}, \quad \mu=1,2, \ldots, \delta(m, n)
$$

and the result follows.

If we let $z=w$ in the previous theorem we get the Pythagorian identity

$$
\frac{\langle z, z\rangle^{m+n}}{m ! n !}=\sum_{\mu=1}^{\delta(q, m, n)} \frac{f_{\mu}(z) \overline{g_{\mu}(z)}}{\left[f_{\mu}, g_{\mu}\right]}, \quad z \in \mathbb{C}^{q} .
$$

When $z \in \Omega_{2 q}$, it reduces to

$$
\frac{1}{m ! n !}=\sum_{\mu=1}^{\delta(q, m, n)} \frac{f_{\mu}(z) \overline{g_{\mu}(z)}}{\left[f_{\mu}, g_{\mu}\right]}
$$

If both bases in the previous theorem are equal and orthonormal with respect to $[\cdot, \cdot]$ then we deduce the addition formula

$$
\langle z, w\rangle^{m}\langle w, z\rangle^{n}=m ! n ! \sum_{\mu=1}^{\delta(q, m, n)} f_{\mu}(z) \overline{f_{\mu}(w)}, \quad z, w \in \mathbb{C}^{q} .
$$

This formula has a structure very similar to that of the addition formula for complex spherical harmonics ([2]). Finally, the following extension of (3.1) 
can be proved in a similar manner:

$$
\langle z, u\rangle^{m}\langle v, z\rangle^{n}=m ! n ! \sum_{\mu=1}^{\delta(q, m, n)} \frac{f_{\mu}(z) \overline{g_{\mu}(u, \bar{v})}}{\left[f_{\mu}, g_{\mu}\right]}, \quad z, u, v \in \mathbb{C}^{q} .
$$

Here, $g_{\mu}(u, \bar{v})$ is obtained from $g_{\mu}(u)=g_{\mu}(u, \bar{u})$, substituting $\bar{u}$ by $\bar{v}$.

In our next result, we establish a Funk-Hecke type theorem for elements in the space $\left(\mathbb{P}\left(\mathbb{C}^{q}\right),[\cdot, \cdot]\right)$.

Theorem 3.2. Let $f$ be an element of $\mathbb{P}_{m, n}\left(\mathbb{C}^{q}\right)$ and $g$ an element of $\mathbb{P}(\mathbb{C})$. Then, for each $w \in \mathbb{C}^{q}$, the map $z \in \mathbb{C}^{q} \mapsto g(\langle z, w\rangle)$ belong to $\mathbb{P}\left(\mathbb{C}^{q}\right)$. In addition, there exists a nonnegative constant $\lambda$, depending on $m$ and $n$, such that

$$
[g(\langle\cdot, w\rangle), f]=\lambda \overline{f(w)}, \quad w \in \mathbb{C}^{q}
$$

Proof. For each pair $(k, l)$, we will denote by $\left\{g_{k, l}^{\mu}: \mu=1,2, \ldots, \delta(q, k, l)\right\}$ an orthonormal basis for $\left(\mathbb{P}_{k, l}\left(\mathbb{C}^{q}\right),[\cdot, \cdot]\right)$. Assume $g$ has degree $\alpha$ in $z$ and degree $\beta$ in $\bar{z}$. Recalling Theorem 3.1, we can write

$$
g(\langle z, w\rangle)=\sum_{k=0}^{\alpha} \sum_{l=0}^{\beta} \sum_{\mu=1}^{\delta(q, k, l)} k ! l ! g_{k, l}^{\mu}(z) \overline{g_{k, l}^{\mu}(w)}, \quad z, w \in \mathbb{C}^{q} .
$$

We can find complex numbers $a_{j}$ such that

$$
f=\sum_{j=1}^{\delta(q, m, n)} a_{j} g_{m, n}^{j}
$$

It follows that

$$
\begin{aligned}
{[g(\langle\cdot, w\rangle), f] } & =\sum_{j=1}^{\delta(q, m, n)} \sum_{k=0}^{\alpha} \sum_{l=0}^{\beta} \sum_{\mu=1}^{\delta(q, k, l)} k ! l ! \overline{a_{j}} \overline{g_{k, l}^{\mu}(w)}\left[g_{k, l}^{\mu}, g_{m, n}^{j}\right] \\
& =\sum_{j=1}^{\delta(q, m, n)} \sum_{k=0}^{\alpha} \sum_{l=0}^{\beta} \sum_{\mu=1}^{\delta(q, k, l)} k ! l ! \overline{a_{j}} \overline{g_{k, l}^{\mu}(w)} \delta_{k m} \delta_{l n} \delta_{\mu j}, \quad w \in \mathbb{C}^{q} .
\end{aligned}
$$

Thus,

$$
[g(\langle\cdot, w\rangle), f]= \begin{cases}m ! n ! \overline{f(w)}, & \alpha \geq m \text { and } \beta \geq n \\ 0, & \text { otherwise, }\end{cases}
$$

completing the proof of the theorem.

Corollary 3.3. The following formula holds

$$
\left[\langle\cdot, w\rangle^{m}\langle w, \cdot\rangle^{n},\langle\cdot, \zeta\rangle^{m}\langle\zeta, \cdot\rangle^{n}\right]=m ! n !\langle\zeta, w\rangle^{m}\langle w, \zeta\rangle^{n}, \quad w, \zeta \in \mathbb{C}^{q} .
$$


The following theorem is a converse of Theorem 3.1.

Theorem 3.4. Let $\left\{f_{\mu}: \mu=1,2, \ldots, \delta(q, m, n)\right\}$ be a linearly independent subset of $\mathbb{P}_{m, n}\left(\mathbb{C}^{q}\right)$. Assume there is a subset $\left\{g_{\mu}: \mu=1,2, \ldots, \delta(q, m, n)\right\}$ of $\mathbb{P}\left(\mathbb{C}^{q}\right)$ such that $\left[f_{\mu}, g_{\mu}\right] \neq 0, \mu=1,2, \ldots, \delta(q, m, n)$ and

$$
\langle z, w\rangle^{m}\langle w, z\rangle^{n}=m ! n ! \sum_{\mu=1}^{\delta(q, m, n)} \frac{f_{\mu}(z) \overline{g_{\mu}(w)}}{\left[f_{\mu}, g_{\mu}\right]} \quad z, w \in \mathbb{C}^{q} .
$$

Then $\left\{f_{\mu}: \mu=1,2, \ldots, \delta(q, m, n)\right\}$ and $\left\{g_{\mu}: \mu=1,2, \ldots, \delta(q, m, n)\right\}$ are bases for $\mathbb{P}_{m, n}\left(\mathbb{C}^{q}\right)$ satisfying $\left[f_{\mu}, g_{\nu}\right]=0, \mu \neq \nu$.

Proof. The use of (3.15) yields

$$
\begin{aligned}
m ! n ! \sum_{\mu=1}^{\delta(q, m, n)} \frac{f_{\mu}(z) \overline{g_{\mu}(\lambda w)}}{\left[f_{\mu}, g_{\mu}\right]} & =\langle z, \lambda w\rangle^{m}\langle\lambda w, z\rangle^{n} \\
& =\langle\bar{\lambda} z, w\rangle^{m}\langle w, \bar{\lambda} z\rangle^{n} \\
& =m ! n ! \sum_{\mu=1}^{\delta(q, m, n)} \frac{f_{\mu}(\bar{\lambda} z) \overline{g_{\mu}(w)}}{\left[f_{\mu}, g_{\mu}\right]} \\
& =m ! n ! \sum_{\mu=1}^{\delta(q, m, n)} \bar{\lambda}^{m} \lambda^{n} \frac{f_{\mu}(z) \overline{g_{\mu}(w)}}{\left[f_{\mu}, g_{\mu}\right]}, z, w \in \mathbb{C}^{q}, \lambda \in \mathbb{C} .
\end{aligned}
$$

Hence

$$
\sum_{\mu=1}^{\delta(q, m, n)}\left(\overline{g_{\mu}(\lambda w)}-\bar{\lambda}^{m} \lambda^{n} \overline{g_{\mu}(w)}\right) \frac{f_{\mu}(z)}{\left[f_{\mu}, g_{\mu}\right]}=0, \quad z, w \in \mathbb{C}^{q}, \quad \lambda \in \mathbb{C} .
$$

Since the set $\left\{f_{\mu}: \mu=1,2, \ldots, \delta(q, m, n)\right\}$ is linearly independent, it follows that

$$
g_{\mu}(\lambda w)-\lambda^{m} \bar{\lambda}^{n} g_{\mu}(w)=0, \quad w \in \mathbb{C}^{q}, \quad \lambda \in \mathbb{C},
$$

that is, $g_{\mu} \in \mathbb{P}_{m, n}\left(\mathbb{C}^{q}\right), \mu=1,2, \ldots, \delta(q, m, n)$. To conclude the proof we apply Theorem 3.2 and formula (3.15) appropriately to obtain

$$
\begin{gathered}
m ! n ! f_{\nu}(z)=\left[\langle\cdot, z\rangle^{m}\langle z, \cdot\rangle^{n}, \overline{f_{\nu}}\right]=m ! n ! \sum_{\mu=1}^{\delta(q, m, n)} \frac{f_{\mu}(z)\left[f_{\nu}, g_{\mu}\right]}{\left[f_{\mu}, g_{\mu}\right]} \\
\nu=1,2, \ldots, \delta(q, m, n) .
\end{gathered}
$$

The linear independence hypothesis allows us to conclude that $\left[f_{\nu}, g_{\mu}\right]=0$, $\mu \neq \nu$. 
Corollary 3.5. If a linearly independent subset $\left\{f_{\mu}: \mu=1,2, \ldots\right.$, $\delta(q, m, n)\}$ of $\mathbb{P}_{m, n}\left(\mathbb{C}^{q}\right)$ satisfies

$$
\langle z, w\rangle^{m}\langle w, z\rangle^{n}=m ! n ! \sum_{\mu=1}^{\delta(q, m, n)} f_{\mu}(z) \overline{f_{\mu}(w)}, \quad z, w \in \mathbb{C}^{q},
$$

then it is orthonormal with respect to $[\cdot, \cdot]$.

Proof. It suffices to observe that, under the given hypotheses, the denominator in the sum on the right-hand side of the last equation in the proof of Theorem 3.4 disappears.

\section{Generating bases}

This section presents a method to construct bases for the space $\mathcal{H}_{m, n}\left(\Omega_{2 q}\right)$. The method is inductive over the dimension of the sphere, that is, it presupposes the knowledge of a basis for $\mathcal{H}_{m, n}\left(\Omega_{2 q-2}\right)$. We begin with a technical lemma that exhibits a very special kernel in $\mathbb{H}_{m, n}\left(\mathbb{C}^{q}\right)$. As we said before, the idea behind the use of this kernel comes from the proof of Theorem 5.1 in [3].

For a fixed $q_{1} \in\{1,2, \ldots, q\}$ we will employ the decomposition $\mathbb{C}^{q}=$ $W^{q_{1}} \oplus V^{q-q_{1}}$, where $W^{q_{1}}=\left\{z \in \mathbb{C}^{q}: z_{j}=0, j=q_{1}+1, q_{1}+2, \ldots, q\right\}$ and $V^{q-q_{1}}=\left\{z \in \mathbb{C}^{q}: z_{j}=0, j=1,2, \ldots, q_{1}\right\}$.

Lemma 4.1. Let $w \in W^{q_{1}} \cap \Omega_{2 q}$ and $v \in V^{q-q_{1}} \cap \Omega_{2 q}$. Then

$$
G_{m, n}^{w, v}(z):=\langle z, v+w\rangle^{m}\langle v-w, z\rangle^{n}, \quad z \in \mathbb{C}^{q}
$$

is an element of $\mathbb{H}_{m, n}\left(\mathbb{C}^{q}\right)$.

Proof. First observe that

$$
\frac{\partial}{\partial \overline{z_{j}}} G_{m, n}^{w, v}= \begin{cases}-n\langle z, v+w\rangle^{m}\langle v-w, z\rangle^{n-1} w_{j} & j=1,2, \ldots, q_{1} \\ n\langle z, w+v\rangle^{m}\langle v-w, z\rangle^{n-1} v_{j} & j=q_{1}+1, q_{1}+2, \ldots, q .\end{cases}
$$

Next, notice that

$$
\sum_{j=q_{1}+1}^{q} \frac{\partial^{2}}{\partial z_{j} \partial \overline{z_{j}}} G_{m, n}^{w, v}=-\sum_{j=1}^{q_{1}} \frac{\partial^{2}}{\partial z_{j} \partial \overline{z_{j}}} G_{m, n}^{w, v}
$$

It follows that $\Delta_{2 q}\left(G_{m, n}^{w, v}\right)=0$. The homogeneity of $G_{m, n}^{w, v}$ with respect to $z$ and $\bar{z}$ is clear. 
If $q_{1}=q-1$ in the previous lemma then $W^{q-1} \cap \Omega_{2 q}$ is a copy of $\Omega_{2 q-2}$. In other words, elements of $W^{q-1} \cap \Omega_{2 q}$ are of the form $\widehat{w}=(w, 0)$ with $w \in \Omega_{2 q-2}$. Denoting the elements of $\mathbb{C}^{q}$ by $\widehat{z}=\left(z, z_{q}\right), z \in \mathbb{C}^{q-1}$, and taking $v=\varepsilon_{q}=(0,0, \ldots, 0,1)$, the function in the previous lemma takes the form

$$
G_{m, n}^{\widehat{w}, v}(\widehat{z})=\left(\langle z, w\rangle+z_{q}\right)^{m}\left(-\langle w, z\rangle+\overline{z_{q}}\right)^{n} .
$$

From now on, we will adopt the following simplified notation: $G_{m, n}^{w}:=G_{m, n}^{\widehat{w}, v}$. The main result of this section is as follows.

Theorem 4.2. Let $\left\{g_{j}: j=1,2, \ldots, d(q, m, n)\right\}$ be a linearly independent subset of $\bigcup_{k=0}^{m} \bigcup_{l=0}^{n} \mathcal{H}_{k, l}\left(\Omega_{2 q-2}\right)$. Then there exists a subset $\left\{f_{j}: j=\right.$ $1,2, \ldots, d(q, m, n)\}$ of $\mathbb{H}_{m, n}\left(\mathbb{C}^{q}\right)$ such that

$$
G_{m, n}^{w}(\widehat{z})=\sum_{j=1}^{d(q, m, n)} f_{j}(\widehat{z}) g_{j}(w), \quad \widehat{z}=\left(z, z_{q}\right) \in \mathbb{C}^{q}, \quad w \in \Omega_{2 q-2} .
$$

Proof. Initially, we expand the right-hand side of (4.3) to write

$$
G_{m, n}^{w}(\widehat{z})=\sum_{|\alpha|+\mu=m} \frac{m !}{\mu ! \alpha !} z^{\alpha} z_{q}^{\mu} \bar{w}^{\alpha} \sum_{|\beta|+\nu=n} \frac{n !}{\nu ! \beta !}(-w)^{\beta} \bar{z}^{\beta} \bar{z}_{q}^{\nu}, \quad \widehat{z} \in \mathbb{C}^{q} .
$$

Since $|\alpha| \leq m$ and $|\beta| \leq n$, a help of (1.8) allows us to find constants $a_{j}(\alpha, \beta)$ such that

$$
\bar{w}^{\alpha}(-w)^{\beta}=\sum_{j=1}^{d(q, m, n)} a_{j}(\alpha, \beta) g_{j}(w), \quad w \in \Omega_{2 q-2} .
$$

Hence,

$$
\begin{aligned}
& G_{m, n}^{w}(\widehat{z}) \\
& =\sum_{j=1}^{d(q, m, n)}\left(\sum_{|\alpha|+\mu=m} \sum_{|\beta|+\nu=n} a_{j}(\alpha, \beta) \frac{m !}{\mu ! \alpha !} z^{\alpha} z_{q}^{\mu} \frac{n !}{\nu ! \beta !} \bar{z}^{\beta} \bar{z}_{q}^{\nu}\right) g_{j}(w) .
\end{aligned}
$$

We now show that the expression

$$
f_{j}(\widehat{z}):=\sum_{|\alpha|+\mu=m} \sum_{|\beta|+\nu=n} a_{j}(\alpha, \beta) \frac{m !}{\mu ! \alpha !} z^{\alpha} z_{q}^{\mu} \frac{n !}{\nu ! \beta !} \bar{z}^{\beta} \bar{z}_{q}^{\nu},
$$

defines an element of $\mathbb{H}_{m, n}\left(\mathbb{C}^{q}\right)$, for $j=1,2, \ldots, d(q, m, n)$. The homogeneity of $f_{j}$ of degree $m$ with respect to $\widehat{z}$ and of degree $n$ with respect to $\bar{z}$ is obvious. Applying the Laplacian in (4.7) we deduce

$$
0=\Delta_{2 q}\left(G_{m, n}^{w}\right)(\widehat{z})=\sum_{j=1}^{d(q, m, n)} \Delta_{2 q}\left(f_{j}\right)(\widehat{z}) g_{j}(w), \widehat{z} \in \mathbb{C}^{q}, w \in \Omega_{2 q-2} .
$$


The linear independence of the $g_{j}$ implies that $\Delta_{2 q}\left(f_{j}\right)=0$.

The following lemma describes an integral operator that reproduces complex spherical harmonics. It is a complex version of the famous Funk-Hecke formula. A proof for this version can be found in [5] and [7]. In the statement of the lemma, $B[0,1]$ is the closed unit disk in $\mathbb{C}, d \nu_{q}(z)$ is the normalized Lebesgue measure given by

$$
d \nu_{q}(z):=\frac{q-1}{\pi}\left(1-x^{2}-y^{2}\right)^{q-2} d x d y, \quad z=x+i y \in B[0,1],
$$

$L^{p, q}(B[0,1])$ is the class of complex functions that are $p$-integrable in $B[0,1]$ with respect to $\nu_{q}$ and $P_{m, n}^{q-2}$ is the disk polynomial of degree $m+n$ associated with the integer $q-2$.

Lemma 4.3. Let $Y$ be an element of $\mathcal{H}_{m, n}\left(\Omega_{2 q}\right)$, and $K$ an element of $L^{1, q}(B[0,1])$. Then for every $w$ in $\Omega_{2 q}$, the mapping $z \in \Omega_{2 q} \longmapsto$ $K(\langle z, w\rangle) Y(z)$ is in $L^{1}\left(\Omega_{2 q}\right)$ and

$$
\int_{\Omega_{2 q}} K(\langle z, w\rangle) Y(z) d \sigma_{q}(z)=\lambda_{n, m}^{q-2}(K) Y(w), \quad w \in \Omega_{2 q}
$$

in which

$$
\lambda_{n, m}^{q-2}(K):=\frac{2 \pi^{q}}{(q-1) !} \int_{B[0,1]} K(z) \overline{P_{n, m}^{q-2}(z)} d \nu_{q}(z) .
$$

Theorem 4.4. Let $\left\{g_{j}: j=1,2, \ldots, d(q, m, n)\right\}$ be a linearly independent subset of $\bigcup_{k=0}^{m} \bigcup_{l=0}^{n} \mathcal{H}_{k, l}\left(\Omega_{2 q-2}\right)$ and let $\left\{f_{j}: j=1,2, \ldots, d(q, m, n)\right\}$ be as in Theorem 4.2. If the set $\left\{g_{j}: j=1,2, \ldots, d(q, m, n)\right\}$ is orthonormal then $\left\{f_{j}: j=1,2, \ldots, d(q, m, n)\right\}$ is an orthogonal basis for $\left(\mathbb{H}_{m, n}\left(\mathbb{C}^{q}\right),[\cdot, \cdot]\right)$.

Proof. In the first step of the proof we show that $\left[G_{m, n}^{w}, G_{m, n}^{\zeta}\right]=K(\langle\zeta, w\rangle)$, for some function $K$. Indeed, recalling the hat notation introduced in the beginning of the section, we see that

$$
\left[G_{m, n}^{w}, G_{m, n}^{\zeta}\right]=D_{1}^{m}\left[\left(\langle\zeta, z\rangle+\overline{z_{q}}\right)^{m}\right] D_{2}^{n}\left[\left(-\langle z, \zeta\rangle+z_{q}\right)^{n}\right],
$$

in which

$$
D_{1}:=\overline{w_{1}} \frac{\partial}{\partial \overline{z_{1}}}+\overline{w_{2}} \frac{\partial}{\partial \overline{z_{2}}}+\cdots+\overline{w_{q-1}} \frac{\partial}{\partial \overline{z_{q-1}}}+\frac{\partial}{\partial \bar{z}_{q}}
$$

and

$$
D_{2}:=-w_{1} \frac{\partial}{\partial z_{1}}-w_{2} \frac{\partial}{\partial z_{2}}-\cdots-w_{q-1} \frac{\partial}{\partial z_{q-1}}+\frac{\partial}{\partial z_{q}} .
$$

However,

$$
D_{1}^{m}\left(\langle\zeta, z\rangle+\overline{z_{q}}\right)^{m}=m !(\langle\zeta, w\rangle+1)^{m}
$$

and

$$
D_{2}^{n}\left(-\langle z, \zeta\rangle+z_{q}\right)^{n}=n !(\langle w, \zeta\rangle+1)^{n}
$$


so that

$$
\begin{array}{r}
{\left[G_{m, n}^{w}, G_{m, n}^{\zeta}\right]=m ! n !(\langle\zeta, w\rangle+1)^{m}(\langle w, \zeta\rangle+1)^{n}:=K(\langle\zeta, w\rangle),} \\
w, \zeta \in \Omega_{2 q-2} .
\end{array}
$$

Next, we use the previous theorem to deduce that

$$
\left[G_{m, n}^{w}, G_{m, n}^{\zeta}\right]=\sum_{l=1}^{d(q, m, n)} p_{l}(w) \overline{g_{l}(\zeta)}, \quad w, \zeta \in \Omega_{2 q-2},
$$

in which

$$
p_{l}(w)=\sum_{j=1}^{d(q, m, n)}\left[f_{j}, f_{l}\right] g_{j}(w), \quad w \in \Omega_{2 q-2} .
$$

If the $g_{l}$ form an orthonormal set we can apply the previous lemma to obtain

$$
\begin{aligned}
\lambda(j) g_{j}(w)=\int_{\Omega_{2 q-2}} K(\langle\zeta, w\rangle) g_{j}(\zeta) d \sigma_{q-1}(\zeta) & =p_{j}(w), \\
j & =1,2, \ldots, d(q, m, n),
\end{aligned}
$$

in which $\lambda(j)$ is a positive constant depending on $g_{j}$ and $K$. Thus,

$$
\left[G_{m, n}^{w}, G_{m, n}^{\zeta}\right]=\sum_{l=1}^{d(q, m, n)} \lambda(l) g_{l}(w) \overline{g_{l}(\zeta)}, \quad w, \zeta \in \Omega_{2 q-2} .
$$

A comparison with (4.19) yields the relation

$$
\begin{aligned}
\lambda(l) g_{l}(w)= & \sum_{j=1}^{d(q, m, n)}\left[f_{j}, f_{l}\right] g_{j}(w), \\
& w \in \Omega_{2 q-2}, \quad l=1,2, \ldots, d(q, m, n) .
\end{aligned}
$$

It is now evident that $\left[f_{j}, f_{l}\right]=0, j \neq l$ and that $\left[f_{l}, f_{l}\right]=\lambda(l), l=$ $1,2, \ldots, d(q, m, n)$.

Example 4.5. Let $m=n=1$ and $q=3$. Due to Lemma 2.9, the polynomials

$$
\begin{array}{r}
g_{1}(w)=\frac{1}{\sqrt{2} \pi}, \quad g_{2}(w)=\frac{1}{\pi} w_{1}, \quad g_{3}(w)=\frac{1}{\pi} w_{2}, \quad g_{4}(w)=\frac{1}{\pi} \overline{w_{1}}, \\
g_{5}(w)=\frac{1}{\pi} \overline{w_{2}}, \\
g_{6}(w)=\frac{\sqrt{3}}{\pi} w_{1} \overline{w_{2}}, \quad g_{7}(w)=\frac{\sqrt{3}}{\pi} \overline{w_{1}} w_{2} \quad \text { and } \\
g_{8}(w)=\frac{\sqrt{6}}{2 \pi}\left(w_{1} \overline{w_{1}}-w_{2} \overline{w_{2}}\right)
\end{array}
$$


define an orthonormal subset of $\mathcal{H}_{0,0}\left(\Omega_{4}\right) \cup \mathcal{H}_{0,1}\left(\Omega_{4}\right) \cup \mathcal{H}_{1,0}\left(\Omega_{4}\right) \cup \mathcal{H}_{1,1}\left(\Omega_{4}\right)$. The kernel $G_{1,1}^{w}(\widehat{z})$ takes the form

$$
\begin{aligned}
z_{3} \overline{z_{3}}-\overline{z_{1}} z_{3} w_{1}-\overline{z_{2}} z_{3} w_{2}+z_{1} \overline{z_{3}} \overline{w_{1}}+z_{2} \overline{z_{3}} \overline{w_{2}}-\overline{z_{1}} z_{2} w_{1} \overline{w_{2}}-z_{1} \overline{z_{2}} w_{2} \overline{w_{1}} \\
-z_{1} \overline{z_{1}} w_{1} \overline{w_{1}}-z_{2} \overline{z_{2}} w_{2} \overline{w_{2}} .
\end{aligned}
$$

Computing the coefficients $a_{j}(\alpha, \beta)$ in (4.6), here written as $a_{j}(\alpha ; \beta)$, we obtain

$$
\begin{gathered}
a_{1}(0,0 ; 0,0)=\sqrt{2} \pi, a_{1}(1,0 ; 1,0)=-\frac{\sqrt{2} \pi}{2}, a_{8}(1,0 ; 1,0)=-\frac{\pi}{\sqrt{6}} \\
a_{1}(0,1 ; 0,1)=-\frac{\sqrt{2} \pi}{2}, \quad a_{8}(0,1 ; 0,1)=\frac{\pi}{\sqrt{6}}, \quad a_{2}(0,0 ; 1,0)=-\pi, \\
a_{4}(1,0 ; 0,0)=\pi, \quad a_{5}(0,1 ; 0,0)=\pi, \quad a_{6}(0,1 ; 1,0)=-\frac{\pi}{\sqrt{3}} \\
a_{7}(1,0 ; 0,1)=-\frac{\pi}{\sqrt{3}}, \quad a_{3}(0,0 ; 0,1)=-\pi,
\end{gathered}
$$

while all the others equal zero. Looking at (4.8), we encounter

$$
\begin{gathered}
f_{1}(\widehat{z})=\frac{\sqrt{2} \pi}{2}\left(-z_{1} \overline{z_{1}}-z_{2} \overline{z_{2}}+2 z_{3} \overline{z_{3}}\right), \quad f_{2}(\widehat{z})=-\pi z_{3} \overline{z_{1}}, \\
f_{3}(\widehat{z})=-\pi z_{3} \overline{z_{2}}, \\
f_{4}(\widehat{z})=\pi z_{1} \overline{z_{3}}, \quad f_{5}(\widehat{z})=\pi z_{2} \overline{z_{3}}, \quad f_{6}(\widehat{z})=-\frac{\pi}{\sqrt{3}} z_{2} \overline{z_{1}},
\end{gathered}
$$

and

$$
f_{7}(\widehat{z})=-\frac{\pi}{\sqrt{3}} z_{1} \overline{z_{2}}, \quad f_{8}(\widehat{z})=\frac{\pi}{\sqrt{6}}\left(-z_{1} \overline{z_{1}}+z_{2} \overline{z_{2}}\right) .
$$

Theorem 4.4 implies that $\left\{f_{j}: j=1,2, \ldots, 8\right\}$ is an orthogonal basis for $\left(\mathbb{H}_{1,1}\left(\mathbb{C}^{3}\right),[\cdot, \cdot]\right)$. The isomorphism $(2.11)$ provides us with an orthogonal basis for $\mathcal{H}_{1,1}\left(\Omega_{6}\right)$.

Corollary 4.6. Assume the hypotheses in Theorem 4.4. If $\left\{g_{j}: j=\right.$ $1,2, \ldots, d(q, m, n)\}$ is orthonormal then

$$
f_{j}(\widehat{z})=p_{j}\left(z_{q}\right) \overline{g_{j}(z)}, \quad z \in \Omega_{2 q-2}, \quad j=1,2, \ldots, d(q, m, n),
$$

in which $\left\{p_{j}: j=1,2, \ldots, d(q, m, n)\right\}$ is a subset of $\mathbb{P}(\mathbb{C})$. 
Proof. If $\left\{g_{j}: j=1,2, \ldots, d(q, m, n)\right\}$ is orthonormal, we can use (4.4) to deduce

$$
f_{j}(\widehat{z})=\int_{\Omega_{2 q-2}} G_{m, n}^{w}(\widehat{z}) \overline{g_{j}(w)} d \sigma_{q-1}(w), \quad z \in \Omega_{2 q-2} .
$$

Expanding $G_{m, n}^{w}$ in the form

$$
G_{m, n}^{w}(\widehat{z})=\sum_{\mu=0}^{m} \sum_{\nu=0}^{n} \frac{(-1)^{\nu} m ! n !}{\mu ! \nu !(m-\mu) !(n-\nu) !} z_{q}^{m-\mu}{\overline{z_{q}}}^{n-\nu} K_{\mu, \nu}(\langle w, z\rangle),
$$

where $K_{\mu, \nu}(\langle z, w\rangle)=\langle z, w\rangle^{\mu}\langle w, z\rangle^{\nu}$, using Lemma 4.3 and arranging we obtain

$$
\begin{array}{r}
f_{j}(\widehat{z})=\left(\sum_{\mu=0}^{m} \sum_{\nu=0}^{n} \frac{(-1)^{\nu} m ! n !}{\mu ! \nu !(m-\mu) !(n-\nu) !} b_{j}(\mu, \nu) z_{q}^{m-\mu}{\overline{z_{q}}}^{n-\nu}\right) \overline{g_{j}(z)}, \\
z \in \Omega_{2 q-2},
\end{array}
$$

where the $b_{j}(\mu, \nu)$ are constants produced by the Funk-Hecke formula. Defining

$$
p_{j}(z)=\sum_{\mu=0}^{m} \sum_{\nu=0}^{n} \frac{(-1)^{\nu} m ! n !}{\mu ! \nu !(m-\mu) !(n-\nu) !} b_{j}(\mu, \nu) z^{m-\mu} \bar{z}^{n-\nu}, \quad z \in \mathbb{C}
$$

concludes the proof.

Corollary 4.7. Assume the hypotheses in Theorem 4.4. If $\left\{g_{j}: j=\right.$ $1,2, \ldots, d(q, m, n)\}$ is orthonormal then

$$
\begin{aligned}
&(\langle\zeta, w\rangle+1)^{m}(\langle w, \zeta\rangle+1)^{n}=D \sum_{j=1}^{d(q, m, n)}\left\langle f_{j}, f_{j}\right\rangle_{2} g_{j}(w) \overline{g_{j}(\zeta)}, \\
& w, \zeta \in \Omega_{2 q-2},
\end{aligned}
$$

in which $D=(m+n+q-1) !\left(2 \pi^{q} m ! n !\right)^{-1}$.

Proof. First we manipulate the sum in the right-hand side of (4.38) to obtain

$$
\begin{aligned}
& \sum_{\mu=1}^{d(q, m, n)}\left\langle f_{\mu}, f_{\mu}\right\rangle_{2} g_{\mu}(w) \overline{g_{\mu}(\zeta)} \\
= & \sum_{\mu=1}^{d(q, m, n)} \sum_{\nu=1}^{d(q, m, n)}\left(\int_{\Omega_{2 q}} f_{\mu}(\widehat{z}) \overline{f_{\nu}(\widehat{z})} d \sigma_{q}(\widehat{z})\right) g_{\mu}(w) \overline{g_{\nu}(\zeta)}
\end{aligned}
$$




$$
\begin{aligned}
& =\int_{\Omega_{2 q}} \sum_{\mu=1}^{d(q, m, n)} f_{\mu}(\widehat{z}) g_{\mu}(w) \overline{\sum_{\nu=1}^{\overline{d(q, m, n)}} f_{\nu}(\widehat{z}) g_{\nu}(\zeta)} d \sigma_{q}(\widehat{z}) \\
& =\int_{\Omega_{2 q}} G_{m, n}^{w}(\widehat{z}) \overline{G_{m, n}^{\zeta}(\widehat{z})} d \sigma_{q}(\widehat{z}), \quad w, \zeta \in \Omega_{2 q-2} .
\end{aligned}
$$

Recalling Lemma 4.1, Theorem 2.7 and Theorem 2.10, we conclude that

$$
\begin{array}{r}
\sum_{\mu=1}^{d(q, m, n)}\left\langle f_{\mu}, f_{\mu}\right\rangle_{2} g_{\mu}(w) \bar{g}_{\mu}(\zeta)=\frac{2 \pi^{q}}{(m+n+q-1) !}\left[G_{m, n}^{w}, G_{m, n}^{\zeta}\right] \\
w, \zeta \in \Omega_{2 q-2}
\end{array}
$$

Finally, (4.18) reduces (4.39) to

$$
\begin{array}{r}
\sum_{\mu=1}^{d(q, m, n)}\left\langle f_{\mu}, f_{\mu}\right\rangle_{2} g_{\mu}(w) \overline{g_{\mu}(\zeta)}=D^{-1}(\langle\zeta, w\rangle+1)^{m}(\langle w, \zeta\rangle+1)^{n}, \\
w, \zeta \in \Omega_{2 q-2},
\end{array}
$$

with $D$ as described in the statement of the corollary.

By letting $w=\zeta$ in Corollary 4.7 we deduce the following identity

$$
\sum_{\mu=1}^{d(q, m, n)}\left\langle f_{\mu}, f_{\mu}\right\rangle_{2}\left|g_{\mu}(w)\right|^{2}=\frac{2^{m+n+1} \pi^{q} m ! n !}{(m+n+q-1) !}, \quad w \in \Omega_{2 q-2} .
$$

We close this section presenting two independent results, one giving an estimate for the sum $\sum_{\mu=1}^{d(q, m, n)}\left\langle f_{\mu}, f_{\mu}\right\rangle_{2}$ and the other explaining why the construction in Theorem 4.2 preserves bi-orthogonality.

Corollary 4.8. Assume the hypotheses in Theorem 4.4. If $\left\{g_{j}: j=\right.$ $1,2, \ldots, d(q, m, n)\}$ is orthonormal then

$$
\sum_{j=1}^{d(q, m, n)}\left\langle f_{j}, f_{j}\right\rangle_{2} \leq \frac{2^{m+n+2} \pi^{2 q-1}}{(q-1) !(q-2) !} .
$$

Proof. First apply the Cauchy-Schwarz inequality to obtain

$$
\begin{aligned}
G_{m, n}^{w}(\widehat{z}) \overline{G_{m, n}^{w}(\widehat{z})} & \leq\langle\widehat{z}, \widehat{z}\rangle^{m}\langle(w, 1),(w, 1)\rangle^{m}\langle\widehat{z}, \widehat{z}\rangle^{n}\langle(-w, 1),(-w, 1)\rangle^{n} \\
& \leq 2^{m+n}\langle\widehat{z}, \widehat{z}\rangle^{m+n}, \quad \widehat{z} \in \mathbb{C}^{q}, \quad w \in \Omega_{2 q-2} .
\end{aligned}
$$

Integration yields

$$
\int_{\Omega_{2 q}} G_{m, n}^{w}(\widehat{z}) \overline{G_{m, n}^{w}(\widehat{z})} d \sigma_{q}(\widehat{z}) \leq 2^{m+n} \int_{\Omega_{2 q}} d \sigma_{q}(\widehat{z})=\frac{2^{m+n+1} \pi^{q}}{(q-1) !},
$$




$$
w \in \Omega_{2 q-2} .
$$

On the other hand, if $\left\{g_{j}: j=1,2, \ldots, d(q, m, n)\right\}$ is orthonormal, the arguments at beginning of the proof of Corollary 4.7 imply that

$$
\sum_{j=1}^{d(q, m, n)}\left|g_{j}(w)\right|^{2}\left\langle f_{j}, f_{j}\right\rangle_{2} \leq \frac{2^{m+n+1} \pi^{q}}{(q-1) !}, \quad w \in \Omega_{2 q-2} .
$$

Finally,

$$
\begin{aligned}
\sum_{j=1}^{d(q, m, n)}\left\langle f_{j}, f_{j}\right\rangle_{2} & =\sum_{j=1}^{d(q, m, n)}\left\langle f_{j}, f_{j}\right\rangle_{2} \int_{\Omega_{2 q-2}}\left|g_{j}(w)\right|^{2} d \sigma_{q-1}(w) \\
& \leq \frac{2^{m+n+1} \pi^{q}}{(q-1) !} \int_{\Omega_{2 q-2}} d \sigma_{q-1}(w)=\frac{2^{m+n+2} \pi^{2 q-1}}{(q-1) !(q-2) !}
\end{aligned}
$$

completing the proof.

Corollary 4.9. Let $\left\{g_{j}: j=1,2, \ldots, d(q, m, n)\right\}$ and $\left\{g_{j}^{\prime}: j=\right.$ $1,2, \ldots, d(q, m, n)\}$ be orthonormal subsets of $\bigcup_{k=0}^{m} \bigcup_{l=0}^{n} \mathcal{H}_{k, l}\left(\Omega_{2 q-2}\right)$ and let $\left\{f_{j}: j=1,2, \ldots, d(q, m, n)\right\}$ and $\left\{f_{j}^{\prime}: j=1,2, \ldots, d(q, m, n)\right\}$ be the corresponding sets resulting from the use of Theorem 4.2. If $\left\langle g_{j}, g_{k}^{\prime}\right\rangle_{2}=0$, $j \neq k$, then $\left[f_{j}, f_{k}^{\prime}\right]=0, j \neq k$.

Proof. We use Corollary 4.6 to write

$$
f_{j}(\widehat{z})=p_{j}\left(z_{q}\right) \overline{g_{j}(z)}, \quad z \in \Omega_{2 q-2}, \quad j=1,2, \ldots, d(q, m, n),
$$

and

$$
f_{j}^{\prime}(\widehat{z})=p_{j}^{\prime}\left(z_{q}\right) \overline{g_{j}^{\prime}(z)}, \quad z \in \Omega_{2 q-2}, \quad j=1,2, \ldots, d(q, m, n),
$$

in which $\left\{p_{j}: j=1,2, \ldots, d(q, m, n)\right\}$ and $\left\{p_{j}^{\prime}: j=1,2, \ldots, d(q, m, n)\right\}$ are subsets of $\mathbb{P}(\mathbb{C})$. It follows, with a help of Theorem 2.7 , that

$$
\begin{aligned}
{\left[f_{j}, f_{k}^{\prime}\right]_{q} } & =p_{j}\left(\frac{\partial}{\partial \overline{z_{q}}}\right)\left(\overline{p_{k}^{\prime}\left(z_{q}\right)}\right)\left[g_{j}, g_{k}^{\prime}\right]_{q-1} \\
& =\frac{m+n+q-1) !}{2 \pi^{q}} p_{j}\left(\frac{\partial}{\partial \overline{z_{q}}}\right)\left(\overline{p_{k}^{\prime}\left(z_{q}\right)}\right)\left\langle g_{j}, g_{k}^{\prime}\right\rangle_{2} .
\end{aligned}
$$

The conclusion in the statement of the Corollary follows. 


\section{References}

[1] Axler, S., Bourdon, P., Ramey, W., Harmonic Function Theory (2nd edition), Graduate Texts in Mathematics 137, Springer-Verlag, New York, 2001.

[2] Koornwinder, T. H., The addition formula for Jacobi polynomials, II. The Laplace type integral representation and the product formula, Math. Centrum Afd. Toegepaste Wisk., Report TW133 (1972).

[3] Koornwinder, T. H., The addition formula for Jacobi polynomials, III. Completion of the proof, Math. Centrum Afd. Toegepaste Wisk., Report TW135 (1972).

[4] Lang, S., Algebra, Addison-Wesley Publishing Co., Inc., Reading, Mass, 1965.

[5] Menegatto, V. A., Oliveira, C. P., Annihilating properties of convolution operators on complex spheres, Anal. Math. 31 (2005), 13-30.

[6] Müller, C., Analysis of Spherical Symmetries in Euclidean Spaces, Appl. Math. Sci. 129, Springer-Verlag, New York, 1998.

[7] Quinto, E. T., Injectivity of rotation invariant Radon transforms on complex hyperplanes in $\mathbb{C}^{n}$, "Integral geometry" (Brunswick, Maine, 1984), 245-260, Contemp. Math. 63, Amer. Math. Soc., Providence, RI, 1987.

[8] Rudin, W., Function Theory in the Unit Ball of $\mathbb{C}^{n}$, Grundlehren Math. Wiss. 241, Springer-Verlag, New York-Berlin, 1980.

[9] Stein, E. M., Weiss, G., Introduction to Fourier Analysis on Euclidean Spaces, Princeton Math. Ser. 32, Princeton Univ. Press, Princeton, NJ, 1971.

Valdir A. Menegatto

Departamento de MATEMÁtica

ICMC-USP — SÃO CARLOS

Caixa Postal 668

13560-970 SÃO CARLOS SP

BRASIL

E-MAIL: MENEGATT@ICMC.USP.BR
C. P. Oliveira

Universidade Federal de ItAJubÁ

ICE-DMC

Caixa Postal 50

37500-903 ItAJUBÁ MG

BRASIL

E-MAIL: OLIVEIRA@UNIFEI.EDU.BR 